\title{
Pourquoi et pour quoi devient-on intérimaire ?
}

Temporary labour: Precariousness and Attractivity?

\section{Dominique Glaymann}

\section{CpenEdition}

\section{Journals}

Édition électronique

URL : http://journals.openedition.org/travailemploi/1982

DOI : 10.4000/travailemploi.1982

ISSN : 1775-416X

\section{Éditeur}

DARES - Ministère du Travail

\section{Édition imprimée}

Date de publication : 15 juin 2008

Pagination : $33-43$

ISSN : 0224-4365

\section{Référence électronique}

Dominique Glaymann, « Pourquoi et pour quoi devient-on intérimaire? », Travail et Emploi [En ligne],

114 | avril-juin 2008, mis en ligne le 05 novembre 2010, consulté le 07 avril 2021. URL : http:// journals.openedition.org/travailemploi/1982 ; DOI : https://doi.org/10.4000/travailemploi.1982 


\title{
Pourquoi et pour quoi devient-on intérimaire?
}

\author{
Dominique Glaymann (*)
}

\begin{abstract}
L'article décrit d'abord les différentes dimensions de la précarité qui sont fréquemment associées au statut de l'intérimaire: instabilité de revenus, difficulté d'accès aux droits sociaux, insécurité professionnelle et sous-emploi, sous-qualification, déclassement, perte d'image sociale et d'identité professionnelle, alors même que le secteur est en plein essor. Pourquoice statut précaire " d'intermittent de l'emploi et donc du chômage» attire-t-il tant de salariés? À partir d'un matériau empirique original et de nombreux entretiens, l'auteur dégage onze raisons possibles, allant du "non-choix» $\grave{a}$ la décision délibérée, représentatives d'une dialectique entre choix et contrainte assez complexe et évolutive. Elles débouchent sur une typologie en quatre postes qui distingue choix faible, contingent, utilitariste et autonome. Mais les intérimaires qui présentent leur choix comme rationnel ou autonome sont-ils en réalité libres ou contraints? Des objectifs plus personnels sont analysés en valeur et en finalité selon les concepts weberiens, et, par grands types, permettent d'approfondir ces parcours et leurs motivations.
\end{abstract}

Chaque année en France, l'intérim voit passer environ 2 millions de salariés qui occupent 550000 à 650000 emplois en équivalents temps plein. Cette forme d'emploi a connu un essor considérable durant les trente dernières années, ce qui conduit à s'interroger sur son attractivité en dépit des difficultés qu'implique sa précarité : poids de l'intermittence et de l'incertitude, risque de pauvreté et de sous-emploi.

Cet essor de l'emploi intérimaire a été concomitant d'un mouvement par lequel les entreprises de travail temporaire ont progressivement élargi les fonctions qu'elles remplissent au sein du système d'emploi. On est ainsi passé de la stigmatisation assez forte du travail intérimaire des années 1960 à son institutionnalisation notamment marquée par la loi de 1972 (la première à réglementer l'intérim et donc à le reconnaître en tant que forme d'emploi particulière), puis à l'inscription par la loi «Borloo» de janvier 2005 des firmes qui l'organisent au sein du «service public de l'emploi(1)». L'évolution des modes de gestion et de recrutement de la maind'œuvre par les entreprises a fortement contribué à assurer un succès croissant à l'intérim. Ainsi, différents acteurs (entreprises de travail temporaire, entreprises utilisatrices, organisations patronales, intérimaires, syndicats de salariés, État, opérateurs publics de l'emploi) ont participé à l'évolution qui a permis au travail temporaire d'attirer de nombreuses entreprises clientes et des millions de salariés.

(*) LARGOTEC, université Paris-Est, glaymann@univ-paris12.fr (1) Cf. Journal officiel de la République française, $\mathrm{n}^{\circ} 15$, 19 janvier 2005.
Parmi les nombreuses questions que cela pose, nous nous demanderons ici ce que cherchent et trouvent ces salariés qui passent par l'intérim. Cela suppose dans un premier temps de rappeler les effets, et notamment les difficultés, que génère cette forme d'emploi sur la vie au travail et hors travail des intérimaires, sur leur insertion professionnelle, lesquelles devraient plus logiquement pousser à éviter l'intérim qu'à lui donner un caractère attractif. Dans un second temps, nous tenterons, à partir d'une étude menée entre 2000 et 2003 dans seize agences intérimaires (voir encadré 1) de dégager les raisons pour lesquelles certains salariés recourent à cette forme d'emploi en dépit de ses inconvénients notables, puis nous aborderons dans leur dimension plus subjective les objectifs poursuivis au cours du passage par l'intérim.

\section{Un secteur en essor, malgré une précarité et un sous-emploi pesants}

La nature provisoire et discontinue des missions rend l'emploi intérimaire intrinsèquement précaire. Il s'agit d'un emploi discontinu, instable, à la durée incertaine. Sa précarité tient au «caractère décisif $\mathrm{du}$ temps et, plus particulièrement, de l'intermittence, entendue comme discontinuité des temps.» (Cingolani, 2005, p. 22). Les droits et les garanties couvrant les intérimaires ont certes été étendus et diversifiés depuis les années 1970 sous l'effet d'un intense partenariat social, mais ces acquis sociaux ne jouent que le temps des missions, même si une «continuité» relative existe puisque c'est le cumul 


\section{Encadré 1 \\ Méthodologie de la recherche}

Outre une analyse bibliographique et statistique, cette étude (dont nous avons rendu compte dans deux ouvrages parus en 2005 et en 2007) s'appuie sur une enquête menée entre 2000 et 2003 dans seize agences franciliennes de quatre entreprises de travail temporaire. Compte tenu de la très grande concentration du secteur (les quatre principaux groupes réalisent $70 \%$ du chiffre d'affaires du travail temporaire), nous avons choisi de centrer nos recherches dans les grands réseaux sans totalement délaisser le cas des PME de façon à éviter le biais éventuel lié au fonctionnement spécifique des grandes entreprises. Nous avons obtenu l'accord de trois des quatre grands réseaux pour mener ce travail ainsi que d'une PME. Malgré quelques contacts qui n'ont pas débouché sur des entretiens avec des intérimaires, nous avons pu travailler sur un éventail de seize agences dont six situées à Paris et dix dans différentes villes de banlieue couvrant l'industrie (neuf agences), le tertiaire (quatre), le transport (deux) et le BTP (un).

L'enquête de terrain a recouru à plusieurs modalités:

- des entretiens avec une quarantaine de salariés des agences d'intérim (désormais appelées “agences d'emploi ») et de responsables de haut niveau des quatre entreprises de travail temporaire;

- une observation in situ permettant de voir, d'entendre et de "sentir» les relations entre les intérimaires et ceux qui les reçoivent en agence et les envoient en mission;

- un questionnement en deux temps d'un panel représentatif de chacune des seize agences avec d'une part l'interrogation par questionnaire de 127 intérimaires et d'autre part des entretiens semi-directifs de 40 à 60 minutes avec 66 de ces 127 répondants;

- des entretiens avec des responsables et des militants syndicaux de la CFDT, de FO et de la CGT ainsi que des personnes ressources du Syndicat des entreprises de travail temporaire (le SETT devenu Prisme en 2006), de l'ANPE, de l'Unedic, de l'Apec et du FAFTT (Fonds d'assurance formation du travail temporaire).

Notre échantillonnage théorique visait à maximiser la diversité des intérimaires interrogés tant sur les dimensions personnelles (genre, âge, lieu de résidence) que professionnelles (métier, qualification, ancienneté au travail et dans l'intérim). La taille réduite de cet échantillon et le taux de non-réponse ne permettent ni une généralisation qui serait évidemment hâtive, ni un chiffrage précis des différents types identifiés au terme de l'analyse des données.

L'enquête par questionnaire était centrée sur la relation des intérimaires à l'emploi et mêlait des aspects quantitatifs et qualitatifs. Le questionnaire a été adressé à un panel représentatif d'intérimaires des seize agences (environ 400 questionnaires ont été envoyés, près de 130 nous ont été retournés). Un tri opéré sur ces résultats nous a ensuite permis de rencontrer une soixantaine d'intérimaires. Nous avions sollicité à la fin du questionnaire un accord des répondants pour réaliser un entretien éventuel, ceux qui étaient d'accord nous confiant alors un numéro de téléphone auquel nous pouvions les joindre.

Nous avons complété nos observations en les confrontant à ceux des enquêtes de Catherine Faure-Guichard conduite en PACA entre 1995 et 1998, de Gilbert Lefèvre, François Michon et Mouna Viprey menées en 2000 en Îlede-France, en Basse-Normandie, dans le Nord-Pas-de-Calais et en Pays de Loire, et à l'étude de la Dares réalisée en 2000 dans la quasi-totalité des régions et s'appuyant notamment sur l'interview de 224 intérimaires.

Les différents contacts que nous avons conservés nous permettent de suivre les évolutions de l'intérim et de réactualiser cette analyse, y compris au moyen d'entretiens avec des responsables d'agences et avec des intérimaires.

Sur ces bases, et en nous appuyant sur des travaux existants, nous avons tenté de tracer différents profils d'intérimaires en fonction de deux éléments qui sont d'une part les raisons qui ont conduit vers le travail temporaire et d'autre part les objectifs éventuellement poursuivis au cours du passage par l'intérim

des heures travaillées dans toutes les entreprises de travail temporaire qui ouvre droit aux indemnités de chômage ou à l'accès à la formation.

La précarité et les difficultés qu'elle engendre tient non seulement à l'incertitude du lendemain, mais aussi à l'absence de fixité professionnelle, qui touche tant le lieu de travail (un intérimaire change sans cesse d'entreprise, d'établissement, d'atelier, de bureau) que le travail (il doit s'adapter à chaque fois aux machines, aux logiciels, aux méthodes, aux exigences en vigueur) et les relations de travail (la hiérarchie, les collègues, les clients). Il faut faire et refaire ses preuves dans le cadre de contraintes comme de liens formels et informels modifiés en permanence.

La vie en intérim est remplie d'un ensemble de problèmes financiers, matériels et psychologiques. La combinaison de salaires (durant les missions) et d'indemnités de chômage (entre les missions) assure une sécurité minimum à ceux qui ont suffisamment cotisé, mais cela suppose un jonglage permanent, source de stress, sans oublier les tracasseries administratives (Jourdain, 2002). Cette instabilité des revenus se double d'une quasi-impossibilité d'accéder aux divers avantages dont bénéficient les salariés en fixe (progressions de carrière, avantages sociaux gérés par les $\mathrm{CE}$ des entreprises utilisa- 
trices sans parler des droits futurs à la retraite). Les intérimaires sont fortement exposés aux différentes formes de pauvreté («d'existence», «monétaire», «subjective», $c f$. BREUIL-GENIER et al., 2001), notamment les jeunes et les peu qualifiés qui sont majoritaires parmi eux (2). Il faut à la fois gérer son budget et supporter un coût psychologique souvent élevé. Comme l'explique Serge Paugam, la précarité de l'emploi rend «probable la précarité des conditions de vie» (PAUgam, 2000, p. 25).

Le risque de pauvreté (LAïB, 2006) provient d'une autre caractéristique du travail intérimaire, que constitue la fréquence du sous-emploi au sens du Bureau international du travail. Si les intérimaires en mission sont des actifs occupés et non des chômeurs, ils sont rarement en plein-emploi. Ils connaissent souvent ce que le BIT nomme du sous-emploi visible ou invisible (ОІт, 1998): situation d'actifs qui travaillent durant un temps inférieur à leurs souhaits ou en dessous de leur qualification. En majorité, ils travaillent moins longtemps qu'ils ne le souhaitent et ont un revenu du travail irrégulier. Moins d'un quart des intérimaires sont en mission de façon continue et à temps plein sur une année et "près de la moitié des intérimaires dit avoir connu au moins une période pendant laquelle ils souhaitaient travailler mais ne se sont pas vus proposer de mission» (FPETT, 2004). D'après le Prisme, «la durée moyenne d'emploi en intérim est de six mois sur une année.» (PrISME, 2006). Selon la Dares, les 1,8 million d'actifs passés par l'intérim en 1999 avaient « exécuté six missions, ce qui représente trois mois à temps plein car la durée moyenne d'une mission est de deux semaines» (JouRDAIN, 2002), ce qui rejoint d'autres évaluations. Les données disponibles témoignent ainsi d'une forte intermittence de l'emploi en intérim. Nombreux sont les intérimaires dont la vie est scandée par des périodes où ils travaillent (en intérim, en CDD voire «au noir») et d'autres où ils chôment; même s'ils ont des missions à plein-temps, ils sont sur l'année en sous-emploi visible.

Intermittents de l'emploi et donc du chômage, les intérimaires connaissent aussi un sous-emploi invisible fréquent aux effets plus durables. Souvent, les missions ne correspondent pas à leur qualification. Beaucoup de jeunes occupant des postes inférieurs à ce que justifieraient leur formation et leurs diplômes (3) paient un triple prix pour leur réputa-

(2) En 2006, comme les années précédentes, un tiers des intérimaires avaient moins de 25 ans, plus de la moitié moins de 30 ans et les deux tiers moins de 35 ans; $39 \%$ étaient ouvriers non qualifiés, $39,5 \%$ ouvriers qualifiés, $12,4 \%$ employés, $7,2 \%$ professions intermédiaires et 1,9\% étaient cadres (PrISME, 2007 selon des données de la Dares).

(3) Différents travaux (Forgeot, Gautié, 1997; Fourcade, 1992) montrent que ce phénomène de sous-qualification durant la période d'insertion professionnelle est déjà ancien et ne se manifeste pas dans le seul cas de l'intérim. tion d'inexpérience: un déficit de revenu, un faible intérêt en contenu de travail et une perte d'opportunité en terme d'expérimentation et d'apprentissage. Le même problème se pose pour nombre de seniors et de femmes. Plus généralement, les salariés les moins diplômés, très nombreux en intérim, sont fréquemment relégués dans des emplois peu qualifiés et mal rémunérés, même lorsque leur expérience professionnelle (que l'on décrète peu transférable sans l'avoir forcément vérifié) justifierait qu'on leur offre mieux en missions comme en formations. La polarisation des missions intérimaires sur des emplois peu ou non qualifiés ne correspond ni à l'expérience professionnelle des plus âgés, ni au profil des intérimaires juniors qui sont, comme dans toute la population, plus diplômés que leurs aînés. Au vu de nos résultats, beaucoup d'intérimaires semblent recrutés sur des postes délibérément sous-qualifiés. Or, un déclassement professionnel fréquent chez les jeunes ( $c f$. NAUZe-Fichet, Tomasini, 2005) a, dans le cas de l'intérim, des effets potentiellement durables pour deux raisons. D'une part, les missions sur des postes de ce type qui s'accumulent sur les curriculum vitae dégradent la réputation professionnelle et font peser des risques de sous-qualification lors des embauches ultérieures. D'autre part, un intérimaire recruté au terme d'une mission a peu de chances de se voir promu à l'embauche. Il risque donc de travailler longtemps au-dessous de son niveau de qualification avec comme conséquences un revenu et une productivité inférieurs à ce que permettrait sa formation. Même quand l'intérim débouche sur un emploi plus durable (CDD) ou plus stable (CDI), il ne sert donc pas toujours de tremplin vers un emploi de qualité, même s'il est très difficile d'identifier et surtout de mesurer un déclassement spécifique des salariés passés par l'intérim.

La précarité dégrade en outre l'image sociale et l'identité professionnelle des intérimaires: ils expérimentent diverses stigmatisations véhiculées par les décisions d'interlocuteurs qui les regardent comme responsables de leur intermittence dans l'emploi (bâilleurs, responsables bancaires, agents de l'ANPE, collègues, et même parents ou amis). Les difficultés pour se loger ou obtenir un emprunt reviennent comme un leitmotiv dans les témoignages. L'ostracisme ressenti par nombre d'intérimaires que nous avons rencontrés se traduit aussi par la nature des tâches qu'on leur confie dans le travail et qui constituent souvent le «sale boulot» dont les autres ne veulent pas. De ce point de vue, nos résultats diffèrent assez sensiblement de ceux tirés de l'enquête menée par l'Insee en 2004 sur la perception du travail dans le secteur privé, selon lesquels «une majorité des salariés en contrat court déclare se sentir tout aussi intégrée à leur entreprise que ceux embauchés en contrat à durée indéterminée (CDI). Leur travail leur paraît tout aussi intéressant...» (FABRE, De Riccardis, 2007, p. 1). Au-delà d'un aspect relationnel souvent médiocre («on n'est 
pas un employé comme les autres. Un intérimaire, ce n'est pas un collègue» résume un sentiment très fréquent), l'impossibilité de s'investir pleinement dans son travail atteint l'identité professionnelle des intérimaires et heurte souvent leur éthique personnelle, leur volonté de montrer leurs capacités et leur fierté de travailleur. ( «Ce qui me pèse le plus, c'est surtout le fait de ne pas pouvoir m'impliquer»est une autre remarque fréquemment entendue).

\section{Un essor qui questionne}

Comme le souligne Richard Sennett, l'instabilité professionnelle engendre un problème de civilisation: "Comment un être humain peut-il se forger une identité et se construire un itinéraire dans une société faite d'épisodes et de fragments? Dans la nouvelle économie, l'expérience dominante est celle de la dérive de lieu en lieu, de job en job.» (SENNETT, 2000, p. 31). Cela nous a conduit à nous demander ce qui explique l'essor de l'intérim et l'attraction qu'il exerce auprès d'un nombre croissant de salariés, en cherchant à identifier les raisons qui expliquent que des salariés toujours plus nombreux recourent à cette forme d'emploi et à comprendre s'ils poursuivent à cette occasion des objectifs précis et, dans l'affirmative, quels sont ces objectifs.

Les raisons sont à chercher à la fois du côté de l'emploi intérimaire et de l'emploi en général. Le premier élément notable est la capacité de l'emploi intérimaire à proposer bien souvent une solution temporaire à ceux qui ne trouvent pas d'autre emploi. La réputation d'efficacité des agences d'intérim repose évidemment sur cette réalité et leur supériorité vis-à-vis de l'ANPE s'explique avant tout par le fait qu'elles n'inscrivent dans leur fichier que les salariés qu'elles pensent pouvoir missionner (après les avoir testés), alors que les agences locales de l'emploi doivent trouver une solution à tous les demandeurs d'emploi. De ce point de vue, on doit constater la simultanéité de l'essor du travail temporaire (nombre de missions multiplié par cinq entre le milieu des années 1970 et le milieu des années 2000) et de la montée d'un chômage de masse devenu persistant (nombre de chômeurs multiplié par quatre durant la même période).

Mais le chômage n'explique pas tout. L'essor quantitatif de l'emploi intérimaire est corrélé d'une part à l'élargissement progressif des fonctions remplies par les entreprises de travail temporaire et d'autre part à leur légitimation parallèle au sein d'un système d'emploi. Le travail intérimaire est passé d'une très forte stigmatisation dans les années 1960 (CAIRE, 1993) à son institutionnalisation progressive notamment marquée par la loi de 1972 (la première à réglementer l'intérim en France en le reconnaissant comme forme d'emploi particulière), puis par la loi «Borloo» de janvier 2005 qui fait des entreprises de travail temporaire des acteurs du «service public de l'emploi». L'évolution des modes de gestion et de recrutement de la maind'œuvre par les entreprises en quête de flexibilité dans un contexte de sous-emploi chronique a grandement contribué à assurer un succès croissant à l'intérim: le secteur s'est évertué à multiplier ses offres de service tout en menant un intense lobbying auprès des pouvoirs publics et en développant un partenariat avec les syndicats, pour obtenir une légitimité encore contestée au début des années 1990. L'image de souplesse et de liberté constitue aussi un argument de vente à l'égard des salariés, notamment jeunes, pour qui l'engagement durable dans une entreprise apparaît moins crédible, peut-être même moins souhaitable qu'auparavant.

Graphique no 1 : L'évolution du nombre de missions d'intérim (en ETP) en France depuis 1975

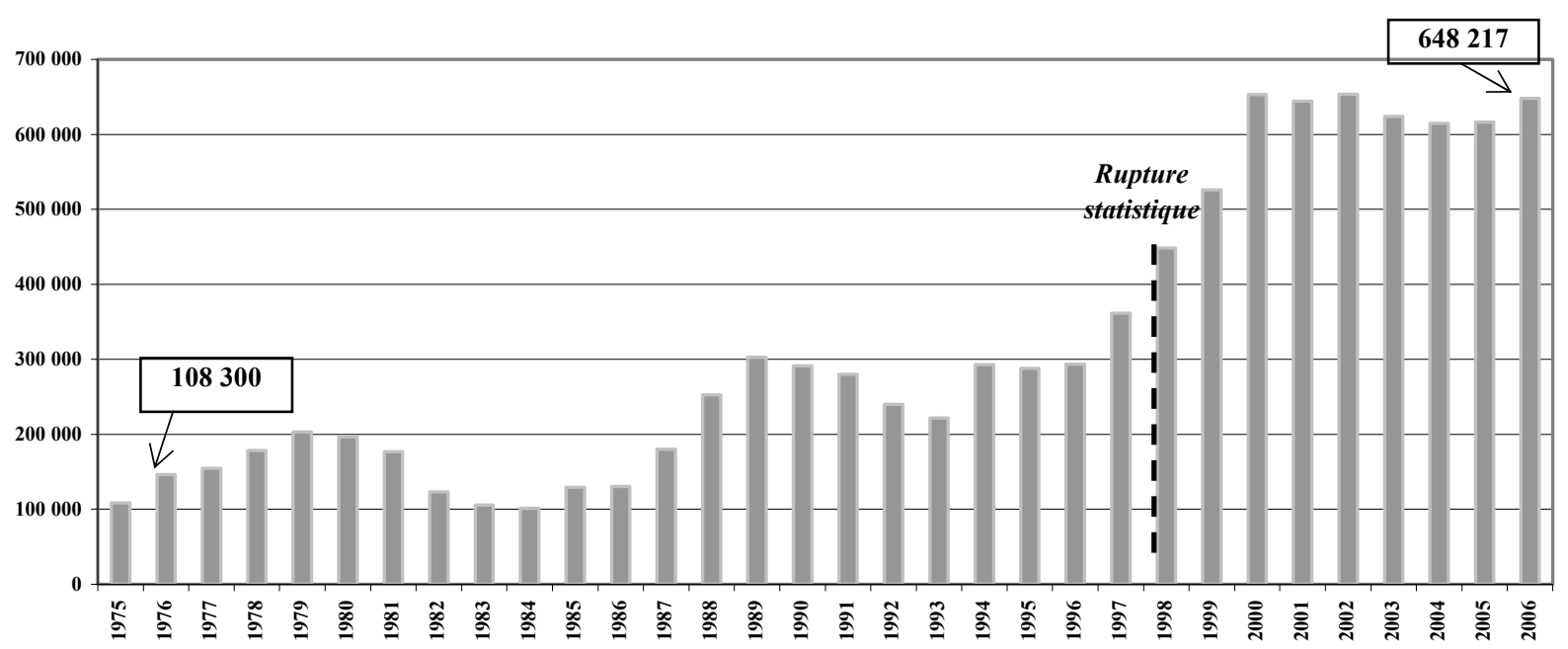

Moyennes annuelles calculées sur la base des données de l’Unedic.

Source: Unedic, Statistiques mensuelles de l'emploi dans l'intérim en données CVS, 2007. 
L'intérim assure désormais différentes «transitions professionnelles» (SCHMID, 1995): transition entre formation et emploi stable, entre chômage et emploi stable, entre inactivité et emploi stable, voire entre emploi et retraite. La très grande part de jeunes parmi les intérimaires montre le rôle que joue l'intérim dans des parcours de primo-insertion professionnelle de plus en plus hachés (CORDELIER, 2006) et incluant un passage par de l'emploi précaire («Plus d'un jeune actif sur cinq occupe un emploi temporaire. Pour nombre d'entre eux, passer par un emploi temporaire constitue une étape incontournable de l'insertion professionnelle», GIVORD, 2006). À l'heure où l'on débat abondamment de flexisécurité et de sécurisation des parcours professionnels, on peut interroger la nature des transitions que propose le travail temporaire. L'idée souvent entendue selon laquelle l'intérim constituerait un tremplin vers l'emploi stable (CANCÉ, FrÉCHOU, 2003) est confirmée pour nombre de jeunes, mais il faut apporter ici deux précisions. D'abord, le fait que la plupart des intérimaires finissent un jour ou l'autre par trouver un CDI ne démontre pas que l'intérim soit la cause de cette stabilisation ni même qu'il l'ait facilitée. Ensuite, ce parcours n'est pas le destin de tous les intérimaires, il a beaucoup à voir avec la conjoncture, l'état de l'emploi dans le(s) métier(s) exercé(s), les ressources dont chacun dispose (diplômes, appartenance à des réseaux) et aussi la part de la chance, voire du hasard. Comme cela a été observé dans le cas du CDD, si l'intérim «peut s'inscrire dans un parcours d'insertion ou de réinsertion, de nombreux salariés peuvent ne pas sortir de ce type d'emploi et se trouver plus exposés à la précarité et au chômage» (CANCÉ, 2002, p. 32). La très grande incertitude de déboucher sur un emploi durable rend ce passage fort peu sécurisant pour beaucoup des salariés concernés.

Les différents éléments qui précèdent permettent de penser que l'état et les mutations du système d'emploi ont conduit nombre d'intérimaires à opter pour cette forme d'emploi sous l'effet d'une coercition dont ils n'ont pas nécessairement conscience et qui est largement indépendante de leurs préférences et de leur histoire personnelle. Il reste que tous les chômeurs, tous les jeunes en quête d'insertion professionnelle, tous les travailleurs de plus de 50 ans en recherche d'emploi, tous les salariés peu qualifiés, toutes les femmes revenant à l'emploi ne passent pas par l'intérim. Il faut alors chercher à comprendre pourquoi on devient intérimaire, et pour quoi on le reste plus ou moins longtemps (4). Le décide-t-on ou le subit-on? Quels sont les poids

(4) Nous traitons ici des passages par les missions d'intérim (même s'il s'agit parfois de pré-embauche) et non de ce qui concerne les autres activités de formation et de recrutement en CDD ou en CDI que réalisent aussi les agences d'intérim depuis la loi du 19 janvier 2005, activités à propos desquelles nous menons actuellement une recherche spécifique. respectifs des pressions socio-économiques et des choix plus ou moins pensés? Que cherche-t-on et que trouve-t-on dans le travail temporaire? Pourquoi choisit-on ou accepte-t-on cette forme d'emploi en dépit des inconvénients liés à sa précarité?

\section{Les raisons du passage par l'intérim}

La recherche des motifs conduisant à choisir ou à accepter le travail intérimaire nous a amené à identifier une diversité de raisons que nous inscrivons dans une dialectique choix/contrainte à la fois plus complexe et plus évolutive qu'une alternative simple entre ces deux moteurs.

\section{Des choix sous contrainte}

Un premier éclairage est apporté par les réponses recueillies en 2002 par l'institut CSA pour le compte du Fonds professionnel pour l'emploi dans le travail temporaire (FPETT) à la question : «Quelles raisons vous ont amené à travailler en intérim?» (Plusieurs réponses possibles étaient proposées).

Ces réponses ( $c f$. graphique 2 ) montrent un recours à l'intérim largement dû aux difficultés de trouver un emploi (pour «trouver rapidement») et voulu comme un passage provisoire (pour «trouver un emploi permanent», faire des «expériences professionnelles» ou «conserver son employabilité»). Seuls $16 \%$ disent être en intérim pour «ne pas travailler tout le temps». Le choix du travail temporaire pour ses caractéristiques propres ne constitue ainsi qu'une explication très minoritaire.

Les résultats tirés de l'Enquête sur la perception du travail dans le secteur privé, selon le type de contrat (enquête complémentaire à l'enquête «Emploi» 2004 de l'Insee) confirment, voire amplifient la perception tirée des données précédentes. S'agissant des intérimaires, les «raisons principales d'acceptation du contrat de travail actuel» sont les suivantes:

Tableau no 1 : Les raisons de l'acceptation de l'intérim par les salariés selon l'Insee

\begin{tabular}{|l|c|c|}
\hline \multicolumn{1}{|c|}{ En \% } & Oui & Non \\
\hline $\begin{array}{l}\text { Vous ne trouviez pas mieux } \\
\text { et avez besoin d'un revenu rapidement }\end{array}$ & 80 & 20 \\
\hline $\begin{array}{l}\text { Éviter une coupure dans votre vie } \\
\text { professionnelle }\end{array}$ & 64 & 36 \\
\hline Le travail proposé vous plaisait & 62 & 38 \\
\hline Obtenir un CDI à l'issue du contrat & 60 & 39 \\
\hline $\begin{array}{l}\text { Acquérir une expérience profession- } \\
\text { nelle permettant d'obtenir un emploi } \\
\text { dans une autre entreprise }\end{array}$ & 45 & 55 \\
\hline Un bon salaire & 40 & 60 \\
\hline
\end{tabular}

Source: Insee, Enquête sur la perception du travail dans le secteur privé, selon le type de contrat, enquête emploi 2004. 


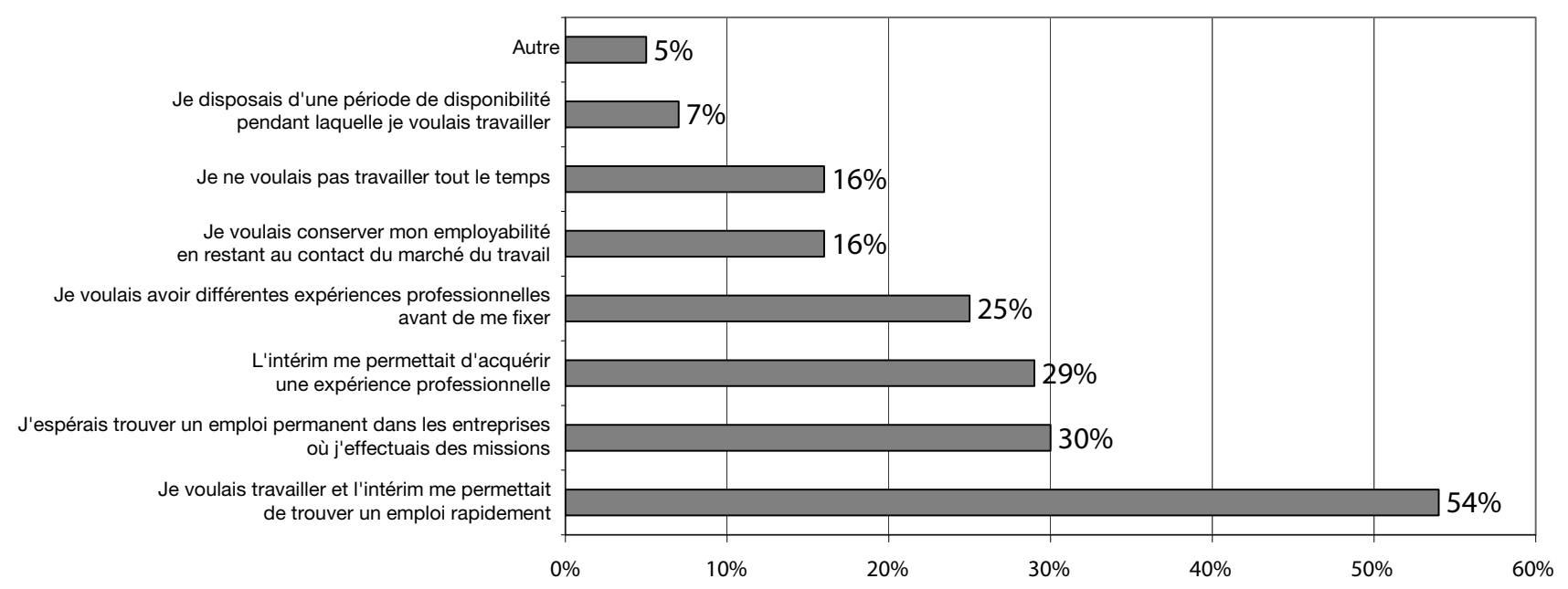

Source: FPETT (Fonds d'assurance formation du travail temporaire) 2003.

À partir d'une enquête menée en 2000, la Dares distinguait cinq types d'explication. Un premier groupe qualifié d'intérim volontaire et provisoire inclut deux sous-groupes: un «intérim d'appoint» ( $14 \%$ de l'échantillon) qui réunit des étudiants, des salariés en formation et quelques-uns en CDD ou en $\mathrm{CDI}$, et un «intérim comme tremplin professionnel» $(23 \%)$. «L'intérim par choix» $(10 \%)$ correspond à un choix volontaire et à long terme. Un «intérim d'attente» $(44 \%)$, dit contraint et provisoire, est constitué de personnes en «emploi provisoire» ou en «intérim en pointillé». Enfin, un «intérim à durée indéterminée» $(9 \%)$ est analysé comme contraint et de longue durée (DARES, 2001). On retrouve en partie la typologie proposée par Catherine FAURE-GUichard (2000) qui distinguait un intérim de profession, un intérim de transition et un intérim d'insertion. Nous proposons d'ajouter à ces trois types ce que nous appellerons un intérim de relégation qui correspond à des «transitions» ratées vers l'emploi stable, qu'il s'agisse de jeunes durablement maintenus dans des emplois sans durée qu'ils alternent avec du chômage ou des formations plus ou moins efficaces, ou de seniors qui ne trouvent pas (ou plus) d'embauche en CDI. Cela montre la très relative part de contrôle de son parcours professionnel et de choix du travail temporaire par les intérimaires, même si cela varie selon leur profil.

\section{Des degrés d'autonomie variables}

À partir de notre enquête, nous avons construit une typologie en fonction de l'autonomie du choix en partant des réponses à une des questions posées dans notre questionnaire: «Pour quelle(s) raison(s) avez-vous décidé de recourir à l'intérim?», thème qui a ensuite été approfondi dans les entretiens.
Nous avions délibérément choisi une question ouverte pour laisser la plus grande liberté de choix de réponse. Notre typologie distingue quatre grands types recouvrant onze degrés croissants en terme d'autonomie dans les raisons. Nous assumons bien entendu le caractère discutable d'un tel classement qui repose sur un regroupement et une interprétation des réponses $(5)$.

Trois précautions s'imposent ici :

- cette typologie s'appuie sur des réponses d'intérimaires (et de chargés de recrutement) qui sont des représentations de la réalité telles que chacun les construit et les raconte au sociologue qui les interroge;

- les choix tout autant que leur interprétation évoluent au fur et à mesure des expériences professionnelles et de la vie des intérimaires;

- aucun choix (même celui dit du «nomadisme») n'est totalement délibéré au sens où cela signifierait l'absence d'effets de contexte. De même, aucune contrainte n'est totalement subie dans la mesure où il y a toujours au moins une acceptation qui conduit à pousser la porte d'une agence ou à signer un contrat de mission.

Le non-choix (degré 1) réunit des recours où la part de décision individuelle est ressentie comme à peu près nulle. Quelques propos typiques l'illustrent: «L'entreprise où je travaille m'a mis directement en relation avec l'agence; la boîte où je faisais mon stage m'a proposé de me prendre en intérim; l'offre que m'a transmis l'ANPE, c'était en intérim, je ne le

(5) «L'élaboration d'une typologie repose sur une interprétation de la réalité et sur le lien entre les phénomènes observés par les enquêtes et certaines des caractéristiques essentielles du milieu particulier, de la condition sociale» (SCHNAPPER, 1999, p. 26). 


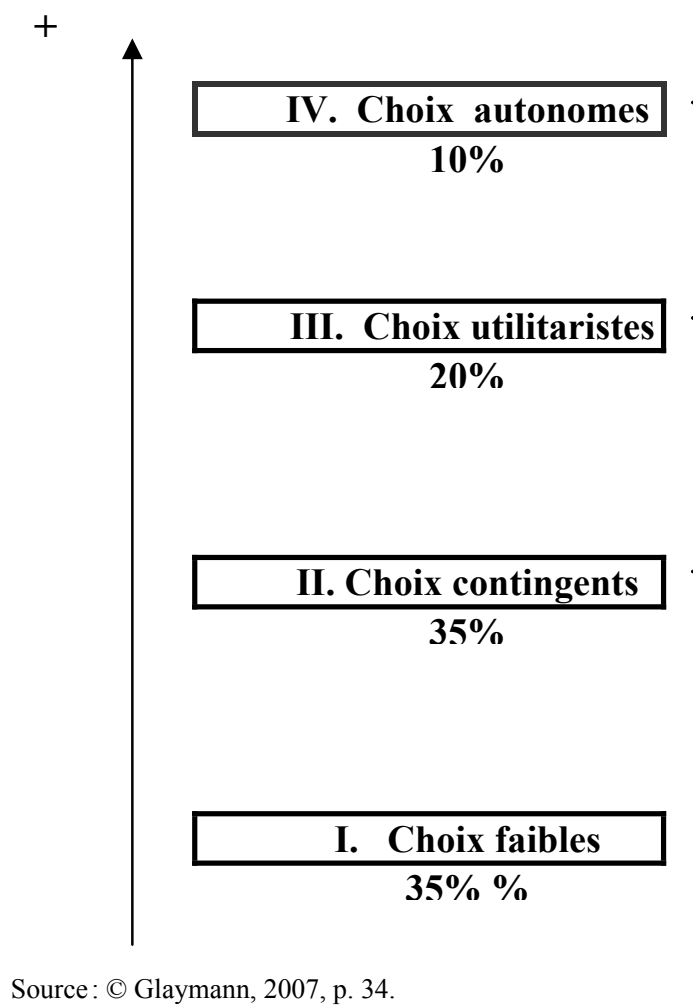

savais pas ». Faute de mieux (degré 2) rassemble des recours totalement subis sous l'effet de contraintes privant de toute marge de manœuvre ou intériorisées comme telles: «par obligation; après avoir déposé des $\mathrm{CV}$ dans des entreprises, je n'ai jamais eu de réponse valable; pas le choix à deux ans de la retraite quand il faut que des sous rentrent pour la petite famille». Au troisième degré pour avoir un emploi et un revenu, on trouve des raisons alimentaires immédiates: «Pour trouver du travail; besoin de travailler, mère isolée, deux enfants à charge; il y a souvent du travail [en intérim] et je n'ai pas de réponses aux demandes dans les entreprises. » L'intérim permet de travailler et d'avoir un salaire en évitant de rester en permanence au chômage. Il paraît difficile ici de parler de choix très actif. Nous évaluons à environ un tiers de notre échantillon(6) les intérimaires classés dans ces trois catégories.

Dans les degrés suivants, les choix d'un intérim comme solution provisoire se font un peu plus volontaires et gagnent en autonomie, depuis ceux qui y recourent en attendant (degré 4) un poste ou un déménagement jusqu'à ceux qui le font lors

(6) Nous présentons à titre indicatif le poids relatif de chacun des quatre types dans notre échantillon. Ces pourcentages ne peuvent pas être extrapolés à l'ensemble des 2 millions de salariés passant chaque année par l'intérim compte tenu de la taille limitée de cet échantillon.

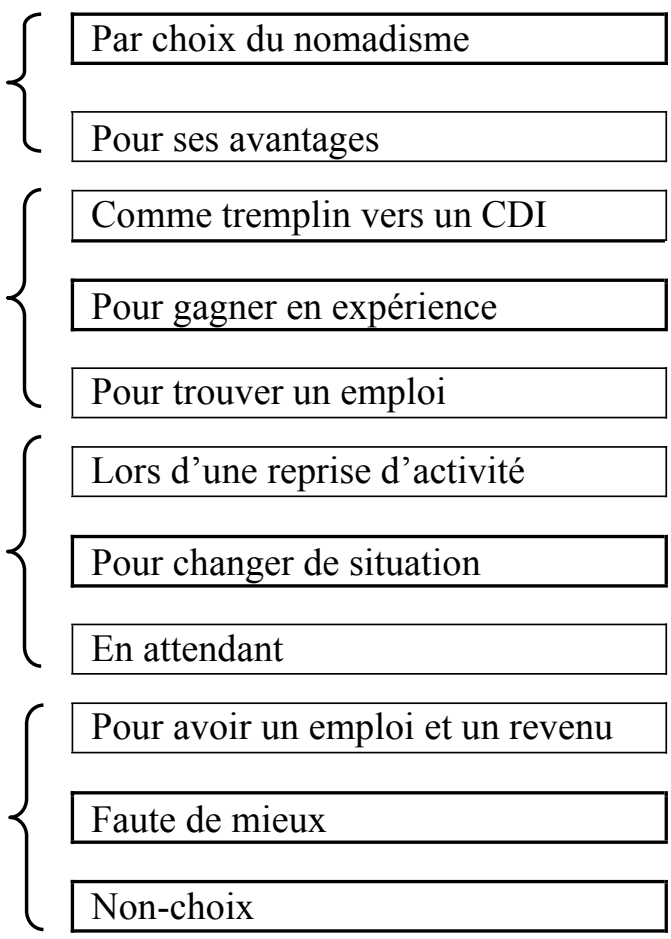

d'une reprise d'activité (degré 6) en passant par ceux qui y passent pour changer de situation (degré 5): «Suite à un accident du travail; suite à un licenciement économique, cela m'a beaucoup aidé à trouver un emploi immédiat, auparavant je faisais divers "petits boulots", avec un niveau BTS tourisme, je n'arrivais pas à exercer dans ma branche, toujours des prétextes à l'embauche; changement de région, l'intérim est le meilleur moyen de se repositionner sur le marché de l'emploi ». Bien que plus contrôlés, les choix restent extrêmement contingents et assez peu délibérés. Ce type réunit ici un autre tiers des intérimaires que nous avons rencontrés.

Au degré 7, pour trouver un emploi, on vise l'efficacité, la rapidité, la simplicité pour chercher un emploi. L'intérim n'est pas choisi pour ce qu'il est, mais comme un instrument auquel on a réfléchi avant d'y recourir: «Rapidité pour trouver une mission, pas de candidature à faire; facilité de l'accès à l'emploi, la recherche étant longue, les démarches nombreuses (ANPE..., les entretiens, rendez-vous, CV...); parce que les embauches sont dures et les entretiens ne sont pas mon point fort». L'intérim pour gagner en expérience (degré 8) est vu comme une étape utile; on trouve notamment là des jeunes confrontés à une insertion difficile: «Pour avoir plus d'expérience professionnelle; pour connaitre des entreprises et avoir de l'expérience; j'ai commencé à 19 ans (durant mes études) lors des vacances scolaires et week-ends pour des raisons de flexibilité et de facilité 
de démarches [...] après mes études, je voulais découvrir différentes entreprises avant de m'investir dans un CDI». Le tremplin vers un CDI (degré 9) correspond à un intérim servant de solution pour trouver un CDI mais aussi pour tester les entreprises: «Cela me permet de me rendre dans les entreprises et de faire mes preuves, ce qui peut se finir par un CDI; permet d'intégrer différentes sociétés, de trouver celle qui me plaît vraiment; tremplin pour obtenir un CDI dans une société qui me convienne.» Ici apparaissent des recours utilitaristes par des actifs qui essaient de tirer profit du rôle d'intermédiation de l'emploi joué par les entreprises de travail temporaire. Environ 20\% de notre échantillon peuvent être considérés comme relevant de ce troisième type.

On trouve enfin dans la partie "supérieure» de ce classement, des choix plus actifs chez ceux qui travaillent en intérim pour ses avantages au degré 10 («L'intérim permet de changer d'entreprise, de mission, de rencontrer plus de gens, en plus c'est mieux payé; pour être plus disponible pour ma famille; parce que j'ai d'autres activités; pour le salaire et la diversité des missions») ou par choix du nomadisme (choix 11): «J'aime les missions courtes et le changement; l'intérim pour échapper à la routine et à l'ennui auxquels certains salariés peuvent faire face». À peine $10 \%$ des intérimaires que nous avons croisés correspondent à ces degrés supérieurs d'autonomie.

Dans le système d'emploi tel qu'il fonctionne aujourd'hui en France, ces réponses confirment qu'on devient le plus souvent intérimaire sous la double pression du chômage et des modes de recrutement. Les raisons du passage par le travail temporaire relèvent d'abord de réalités externes même si, à force d'intérioriser ces contraintes sociales, beaucoup parlent de choix et en viennent à afficher un certain nombre d'objectifs poursuivis au cours ou au moyen du passage par l'intérim. Ce qui nous a permis de prolonger l'analyse.

\section{L'affirmation d'objectifs qui donnent du sens pour "faire avec" l'intérim}

À notre sens, un objectif revêt la dimension subjective d'une volonté construite, alors qu'une raison renvoie plutôt à une donnée objective externe qui exerce une coercition plus ou moins forte. On peut avoir différents objectifs en recourant au travail temporaire: réaliser le but immédiat de travailler, mettre à profit l'intérim même si on ne l'a pas choisi, l'inclure dans un parcours que l'on construit ou vivre selon ses envies. Ces objectifs peuvent avoir été prédéfinis autour d'une orientation raisonnée, ils peuvent aussi être pensés au fur et à mesure, ils peuvent encore n'être que des réponses aux contraintes auxquelles il faut bien se soumettre.
Il faut donc être prudent avant de parler de stratégie en tentant d'interpréter des récits dans lesquels il est probable que certains affichent des objectifs qu'ils n'avaient pas a priori. On peut cependant, même dans ce cas, poursuivre un objectif en mobilisant des moyens et en adoptant une posture volontaire sur la base d'une analyse de sa situation et de son parcours. On est alors guidé par une rationalité qui vise à décider de ses actes tout en subissant des contraintes dont on est plus ou moins conscient. Dans tous les cas, les objectifs présentés, même construits au fur et à mesure, même peu stratégiques, aident à supporter la précarité et ses effets en donnant un sens à ce que l'on vit.

\section{Des objectifs divers}

Un intérimaire doté d'objectifs choisit le travail temporaire ou tente d'en profiter s'il n'a pu l'éviter. Sa vision peut n'être qu'immédiate, elle peut être utilitariste (pour aménager un parcours), elle peut s'inscrire dans la durée par goût pour ce type d'emploi et le mode de vie qui va avec. La différence entre un objectif mûrement réfléchi et une réaction à des stimuli extérieurs est ténue: «La frontière entre une activité significative et un comportement (que j'appellerai ici) simplement réactionnel, parce que non associé à un sens visé subjectivement, est absolument flottante.» (WEBER, 1971, p. 4). Les objectifs des intérimaires varient par leur nature, leur durabilité et leur complexité. Notre lecture récuse une analyse qui réduirait les actes individuels à des arbitrages entre coûts et avantages pour atteindre des positions calculées et qui expliquerait le destin de chacun par sa seule capacité à surmonter les difficultés. Mais nous ne pensons pas non plus que les individus, ici les intérimaires, ne seraient que les jouets des événements et des structures. Si le cadre social génère des contraintes (et elles sont très fortes au sein du système d'emploi) qui pèsent de façon déterminante, la plupart des intérimaires que nous avons rencontrés tentent néanmoins de mener leur vie en faisant des choix pour arriver à certaines fins. Ils poursuivent ainsi des objectifs, chacun selon ses ressources et ses capacités à s'en servir, même si leur sens n'est pas toujours postulé. Non seulement la rationalité est limitée, mais elle est souvent obscure.

Certains des objectifs que nous avons repérés sont "professionnels» (travailler vite, trouver un $\mathrm{CDI}$, gagner en expérience, changer de métier ou de secteur), certains sont «existentiels» (gérer son temps, choisir ses missions et ses déplacements), d'autres sont « mixtes» (ils concernent l'emploi et le mode de vie). Les concepts de rationalité en valeur et en finalité construits par Max Weber nous ont aidé à comprendre ces parcours et ces motivations.

\section{Des objectifs rarement stratégiques}

La quasi-totalité des intérimaires du type I (choix faibles), une nette majorité de ceux du type II (choix 
contingents) et une partie du type III (choix utilitaristes) tentent de faire face à des obligations sociales inéluctables: ne travailler en intérim «que» pour avoir un emploi et un revenu, et «simplement» parce qu'on n'a pas trouvé mieux ne forme pas une stratégie. Recourir à l'intérim «seulement» pour subvenir à ses besoins est certes un but, mais cela revient à respecter une norme fondamentale de socialisation et non à poursuivre des objectifs propres, on peut parler de conduite "coutumière (7)». Ainsi, une préparatrice de commandes de 33 ans, intérimaire depuis trois ans, qui s'est inscrite dans une agence parce que «j'en avais marre de chercher du travail [...]. Moi, je suis quelqu'un qui a besoin que ça marche tout de suite. Si ça ne marche pas tout de suite, je laisse tomber» répondait à notre question sur ses objectifs: «Moi, je ne veux plus me prendre la tête à chercher du boulot. Je suis très bien en intérim ». Vous travaillez aussi régulièrement que vous le souhaitez? «Depuis que je suis en intérim, oui. À part les vacances, où je ne travaille pas». C'est vous qui choisissez les dates de vacances? «Non, ce n'est pas moi qui choisis. [...]» Vous vous imaginez intérimaire dans dix ou quinze ans? «Pourquoi pas? Je ne sais pas. Franchement, je n'espère pas, mais...» Pourquoi vous espérez que non? «Trouver quelque chose de fixe, ce serait bien, mais s'il n'y a pas...». On peut ici parler d'objectifs induits qui ne sont pas stratégiques. Une grande majorité d'intérimaires cherchent à avoir un emploi pour gagner leur vie et pour acquérir un statut «normal». Ils n'ont pas choisi d'être intérimaires même s'ils ont décidé un jour de le devenir. Cela ne les empêche pas d'essayer d'exploiter ce passage pour améliorer leur position dans l'emploi, se fixant ainsi des objectifs qui dépassent l'emploi provisoire qu'ils recherchaient en entrant dans une agence d'intérim. Certains finissent même parfois par définir des objectifs de moyen ou de long terme après avoir analysé les atouts dont ils disposent ou dont ils doivent se doter. En visant à convaincre chacun qu'il lui revient de tirer son épingle du jeu en améliorant ses savoirs, ses savoir-faire et ses «savoir-être», l'action du personnel des agences les pousse d'ailleurs dans ce sens.

Une partie des intérimaires du type III (choix utilitaristes) et la quasi-totalité du type IV (choix autonomes) poursuivent des objectifs plus rationalisés: ils prennent des initiatives, font des choix et ont une certaine maîtrise de leur trajectoire. Ces intérimaires analysent l'intermédiation des agences comme une aide pour se faire connaître, améliorer leur image aux yeux des recruteurs et trouver un

(7) Loin de révéler «la fin du travail», la crise de l'emploi et les difficultés récurrentes de l'intégration confirment plutôt une centralité du travail encore très présente et prégnante tant dans les pratiques d'intégration et de construction des liens sociaux que dans le système de valeurs et de normes qui reste ancré au cœur de notre société. emploi. Même si c'est aussi le fonctionnement de l'emploi qui explique leur présence en intérim, ils diffèrent de ceux qui y ont été «enrôlés» pour les mêmes raisons, car ils ont rationalisé leur parcours et tentent de retourner la contrainte à leur profit. Plutôt que de multiplier les $\mathrm{CV}$, les rendez-vous à l'ANPE, les entretiens d'embauche, ils préfèrent gagner la confiance d'un(e) chargé(e) de recrutement d'une agence d'intérim puis enchaîner les missions, améliorer leur employabilité pour se rapprocher du CDI qu'ils visent plus ou moins impatiemment. Nous parlons ici d'employabilité au sens de Bernard Gazier qui la définit comme non «seulement la capacité à trouver un emploi, mais celle de construire une carrière autonome, la capacité à obtenir et à conserver un emploi» (GAZIER, 2003, p. 99) en soulignant le poids du contexte et des interactions. Les récits «positifs» que présentent beaucoup d'intérimaires ne doivent pas masquer les difficultés rencontrées (disponibilité totale exigée, périodes sans mission parfois longues et répétées, sales boulots...). Ici encore, le recours à l'intérim sert d'abord à pallier les difficultés liées au chômage et aux obstacles à l'embauche, mais l'intérim permet aussi de faire des expériences, de compléter une formation et d'alimenter des CV. Ces stratégies réfèrent à une certaine rationalité en finalité. Ainsi, Freddy, tuyauteur de 40 ans, en intérim depuis deux ans après avoir subi un licenciement pour motif économique, explique: "Il faut que je me fasse embaucher en CDI pour pouvoir changer de logement [mais] j'ai refusé d'être embauché dans l'entreprise où je travaille actuellement à cause du climat. [...] Les relations patron/employés sont mauvaises, on n'est pas respecté». Alors? «Je veux trouver le "bon" CDI [et en attendant] je continue en intérim, jusqu'à ce que je trouve». C'est un moyen de voir comment ça se passe avant une éventuelle embauche? «Oui, je peux tester mon patron». Avant embauche? «Oui, c'est ça».

Une part minime des intérimaires de notre type IV (choix autonomes) a accepté ou même décidé de travailler sous ce statut pour avoir une latitude dans le choix des emplois et dans l'organisation du temps. Au-delà de la seule sphère professionnelle, leurs objectifs concernent des choix de vie. Ils aspirent à partager à leur gré le temps travaillé et le temps libre en échappant à la monotonie et à la contrainte qu'implique un contrat durable. Ils tentent de fuir les contraintes du CDI en aménageant leur parcours. Leur conduite est un mélange de rationalités en finalité et en valeur. Ils rêvent de liberté, d'arbitrage entre travail et non-travail. Ces intérimaires ont souvent beaucoup d'illusions sur la liberté comme sur le revenu que le travail temporaire leur procure. La domination de l'idéologie prônant la liberté individuelle comme valeur centrale les aveugle largement sur ce qu'ils «gagnent» en pensant gérer leur «capital humain» et sur ce qu'ils perdent à la déstructuration des collectifs de travail, 
mais ils adhèrent néanmoins à cette logique et pensent maîtriser leur destin. Kemal, 25 ans, manutentionnaire, en intérim depuis cinq ans, explique ainsi: «Dans l'intérim, ce qui me plaît déjà, c'est qu'on travaille trois semaines et c'est équivalent à cinq semaines. Ça dépend de la mission, mais en général c'est très bien payé. [...] avec les indemnités et les congés payés, les primes de déplacement, et puis si on travaille avec des boîtes qui ont des risques, il y a des primes de risque, tout ça, ça peut faire beaucoup. [...] Moi, si le patron me fait ch..., je lui dis : "Vas-y, tu me fais ch...! “"» (La suite de l'entretien montre que ce cas de figure est en fait très théorique, mais il vit tout de même avec et grâce à cette vision des choses).

Un dernier type d'objectifs concerne les intérimaires qui veulent fuir la subordination salariale grâce au travail temporaire en adoptant une espèce de «nomadisme» comparable à celui des anciens compagnons, ou à la façon des «Sublimes (8)». L'intérim leur assure un travail et un revenu, il leur permet de gérer leur calendrier et de choisir les missions, les tâches et les entreprises. Ils rejettent l'engagement auprès d'un employeur avec qui se noueraient des obligations réciproques durables même s'ils recherchent et trouvent souvent une fidélité avec une entreprise de travail temporaire, voire une agence pour ceux qui restent dans le même bassin d'emploi. «Moi, il faut que je sois bien dans mon travail. Si ça ne me plaît pas, ce n'est pas la peine, hein! C'est tout, ça s'arrête là. Je suis habitué à travailler tout seul, à gérer mes affaires moi-même. Moi, dans le travail, ce qui me plait, c'est d'être très indépendant» raconte Pascal, électricien hautement qualifié, en intérim depuis vingt-sept ans. Se sentant libres de leurs engagements qui ne sont que ponctuels, ces intérimaires ne ressentent pas leur subordination avec la même acuité que «les fixes» dont ils se distinguent délibérément: «Ce n'est pas n'importe qui, qui reste plus de vingt-cinq ans en intérim, il ne faut pas croire, moi je vous le dis!» dit fièrement Pascal. Cette catégorie d'intérimaires révèle la persistance voire la résurgence d'un refus de la condition salariale présente depuis les débuts du salariat. C'est sans doute aussi un symptôme de l'évolution en cours des conventions touchant le travail et l'emploi. Mais cette dernière catégorie est statistiquement très marginale.

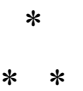

(8) Cf. Gazier (2003) et Poulot D., (1980), Le Sublime ou l'ouvrier tel qu'il est en 1870, Paris, Maspero (première éd. en 1872).

- 42 • Travail et Emploi $n^{\circ} 114 \cdot$ Avril-juin 2008
Notre enquête nous a montré qu'un nombre significatif d'intérimaires dit utiliser l'intérim à des fins personnelles. Évidemment influencées par les discours ambiants, ces visions subjectives participent de la réalité sociale et de ses pesanteurs idéologiques. Beaucoup disent inscrire leur passage par l'intérim dans un parcours qu'ils gèrent au moins en partie et au cours duquel ils visent et parfois atteignent différents buts, même si bien peu peuvent se fixer des objectifs à moyen et long termes et gérer leur trajectoire. Pour notre part, nous classons les règles du jeu en matière d'emploi dans la catégorie des faits sociaux «doués d'un pouvoir de coercition en vertu duquel ils s'imposent» et qui «existent en dehors des consciences individuelles» (DuRKHEIM, 1990). Néanmoins, beaucoup d'intérimaires affichent des objectifs, même s'ils s'inscrivent le plus souvent dans le court terme, même s'ils sont revus à mesure des événements extérieurs et des choix des autres acteurs. Ces représentations doivent être prises en compte si l'on refuse de considérer ces personnes « comme de simples supports de positions sociales dont les discours et les pratiques ne seraient que des conséquences mécaniques et programmées » (DuBet, 2007, p. 24). 


\section{Bibliographie}

Breuil-Genier P., Ponthieux S., Zoyem J.-P. (2001), «Profils sur le marché du travail et caractéristiques familiales des actifs pauvres», Économie et Statistique, $\mathrm{n}^{\circ}$ 349-350, Insee, pp. 99-124.

CAIRE G. (1993), Le Travail intérimaire, Paris, PUF.

CANCÉ R. (2002), «Travailler en contrat à durée déterminée: entre précarité contrainte, espoir d'embauche et parcours volontaire», Travail et Emploi, no 82, pp. 29-44.

CANCÉ R., FrÉCHOU H. (2003), «Les contrats courts: source d'instabilités mais aussi tremplin vers l'emploi permanent», Premières Informations, Premières Synthèses, no 30.2, avril.

Cingolani P. (2005), La Précarité, Paris, PUF.

Cordelier C. (2006), «De 1977 à 2002, l'emploi des jeunes salariés est de plus en plus découpé par des interruptions », Insee Première, no 1104, octobre.

DAREs (2001), Enquête sur le devenir des intérimaires, $1^{r e}$ et $2^{e}$ cohorte. Résultats de synthèse, mars.

DARES (2007), «L'intérim en 2006: une croissance toujours soutenue», Premières Informations, Premières Synthèses, $\mathrm{n}^{\mathrm{o}} 30.2$, juillet.

Dubet F. (2007), L'Expérience sociologique, Paris, éditions La Découverte.

Durkheim É. (1990), Les Règles de la méthode sociologique [1895], Paris, PUF.

FABRE É., De RicCARDis N. (2007), «Les contrats courts vus par les salariés : une précarité de l'emploi qui n'induit pas nécessairement une précarité du travail», Premières Informations, Premières Synthèses, no 12.3, mars.

FAure-Guichard C. (2000), L'Emploi intérimaire, trajectoires et identités, Presses universitaires de Rennes.

FAure-Guichard C. (1999), «Les salariés intérimaires, trajectoires et identités », Travail et Emploi, $\mathrm{n}^{0} 78$.

Forgeot G., Gautié J. (1997), «Insertion professionnelle des jeunes et processus de déclassement», Économie et Statistique, $\mathrm{n}^{\mathrm{o}}$ 304-305, pp. 53-74.

Fourcade B. (1992), «L'évolution des situations d'emploi particulières de 1945 à $1990 »$, Travail et Emploi, no 52, pp. 4-19.

FPETT (2003), Regards des intérimaires... sur l'intérim, étude de l'institut BVA.

FPETT (2004), Regards des intérimaires... sur l'intérim, étude de l'institut BVA.

GAZIER B. (2003), Tous sublimes! Vers un nouveau pleinemploi, Paris, Flammarion.
Givord P. (2005), «Formes particulières d'emploi et insertion des jeunes », Économie et Statistique, no 388 389, pp. 129-143.

Givord P. (2006), «L'insertion des jeunes sur le marché du travail entre 2002 et $2004 »$, Insee Première, no 1061 , janvier.

Glaymann D. (2005), La Vie en intérim, Paris, Fayard.

Glaymann D. (2007), L'Intérim, Paris, La Découverte.

INSEE (2001，2002，2003，2004，2005，2006，2007), Enquêtes annuelles sur l'emploi 2000 à 2006, Insee.

JouRDAIN C. (2002), «Intérimaires, les mondes de l'intérim», dans «Dossier: regards croisés sur les emplois flexibles », Travail et Emploi, no 89, pp. 9-28.

LAÏB N. (2006), «Situation sur le marché du travail et pauvreté monétaire», Études et Résultats, no 499, juin.

LefÈvre G., Michon F., Viprey M. (2002), «Les Stratégies des entreprises de travail temporaire, acteurs incontournables du marché du travail, partenaires experts en ressources humaines », dans «Dossier : regards croisés sur les emplois flexibles», Travail et Emploi, $\mathrm{n}^{\circ}$ 89, pp. 45-64.

Nauze-Fichet É., Tomasini M. (2005), «Parcours des jeunes à la sortie du système éducatif et déclassement salarial», Économie et Statistique, no 388-389, pp. 57-83.

OIT (1998), La mesure du sous-emploi, XVI ${ }^{\mathrm{e}}$ Conférence internationale des statisticiens du travail, Genève.

Paugam S. (2000), Le salarié de la précarité. Les nouvelles formes de l'intégration professionnelle, Paris, PUF.

PRISME (2006), Etre intérimaire, www.prisme.eu, sept.

Prisme (2007), Rapport économique et social, www. prisme.eu

Schmid G. (1995), «Le plein-emploi est-il encore possible? Les marchés du travail transitoires en tant que nouvelle stratégie dans les politiques d'emploi», Travail et Emploi, no 65, 4/95, pp. 5-17.

SCHNAPPER D. (1999), La Compréhension sociologique, Paris, PUF.

SennetT R. (2000), Le Travail sans qualités, les conséquences humaines de la flexibilité, Paris, Albin Michel.

Weber M. (1971), Économie et Société, tome 1 [1921], Paris, Plon. 